\title{
ARTICLE
}

Received 21 Aug 2013 | Accepted 22 Nov 2013 | Published 2 Jan 2014 DOI: 10.1038/ncomms3995

\section{High yield exfoliation of two-dimensional chalcogenides using sodium naphthalenide}

Jian Zheng', Han Zhang', Shaohua Dong', Yanpeng Liu', Chang Tai Nai', Hyeon Suk Shin², Hu Young Jeong ${ }^{2}$, Bo Liu' \& Kian Ping Loh ${ }^{1}$

Transition-metal dichalcogenides like molybdenum disulphide have attracted great interest as two-dimensional materials beyond graphene due to their unique electronic and optical properties. Solution-phase processes can be a viable method for producing printable singlelayer chalcogenides. Molybdenum disulphide can be exfoliated into monolayer flakes using organolithium reduction chemistry; unfortunately, the method is hampered by low yield, submicron flake size and long lithiation time. Here we report a high-yield exfoliation process using lithium, potassium and sodium naphthalenide where an intermediate ternary $\operatorname{Li}_{x} M X_{n}$ crystalline phase ( $X=$ selenium, sulphur, and so on) is produced. Using a two-step expansion and intercalation method, we produce high-quality single-layer molybdenum disulphide sheets with unprecedentedly large flake size, that is up to $400 \mu \mathrm{m}^{2}$. Single-layer dichalcogenide inks prepared by this method may be directly inkjet-printed on a wide range of substrates.

\footnotetext{
${ }^{1}$ Department of Chemistry and Graphene Research Centre, National University of Singapore, 3 Science Drive 3, Singapore 117543, Singapore.

2 Interdisciplinary School of Green Energy and Low Dimensional Carbon Materials Center, UNIST Central Research Facilities (UCRF), Ulsan National Institute of Science and Technology (UNIST), UNIST-gil 50, Ulsan 689-805, Korea. Correspondence and requests for materials should be addressed to K.P.L. (email: chmlohkp@nus.edu.sg).
} 
ost graphene discovery, single-layered transition-metal dichalcogenides (LTMDs), such as $\mathrm{MoS}_{2}$ and $\mathrm{WS}_{2}$, have attracted great attention as next generation twodimensional (2D) materials owing to their large intrinsic bandgap ${ }^{1-13}$, which is particularly attractive in view of the gapless nature of graphene $e^{14-16}$. Single-layer $\mathrm{MoS}_{2}$ has attractive attributes such as a direct bandgap $(1.9 \mathrm{eV})$, large in-plane mobilities $\left(200-500 \mathrm{~cm}^{2} \mathrm{~V}^{-1} \mathrm{~s}^{-1}\right)$, high current on/off ratios (exceeding $10^{8}$ ), as well as remarkable mechanical and optical properties ${ }^{17,18}$. Two-dimensional quantum confinement of carriers can be exploited in conjunction with chemical composition to tune the optoelectronic properties of the metal chalcogenides at the nanoscale. These properties are of great interest for applications in optoelectronic devices such as thin film solar cells, photodetectors, flexible logic circuits and sensors ${ }^{19-23}$.

Transition-metal chalcogenides (TMC) possessing lamellar structures can serve as hosts for the intercalation of a wide variety of electron-donating species ranging from Lewis base to alkali metals ${ }^{24,25}$. One well-known class of intercalatants is the organolithium compounds. $\mathrm{MoS}_{2}$ can be intercalated with lithium to give the reduced $\mathrm{Li}_{\mathrm{x}} \mathrm{MX}$ phase $(\mathrm{X}=\mathrm{Se}, \mathrm{S}$, and so on) with expanded lattice, this can be exfoliated in a second step into single-layer sheets by ultrasound-assisted hydration process $^{26-29}$. However long lithiation time (for example, 3 days at $100^{\circ} \mathrm{C}$ ) is typically needed when $n$-butyl lithium is used as lithiation agent due its inclination to form dimeric, trimeric and higher aggregates, which diffuse slowly into the interlayers in large-sized TMC crystals. Subsequent exfoliation from the lithiated $\mathrm{MoS}_{2}$ suffers a low yield of single-layer flakes, disintegration into submicron-sized flake, formation of metal nanoparticles and precipitation of $\mathrm{Li}_{2} \mathrm{~S}^{5,29}$. This has limited the development of solution-processed LTMDs in most applications that demand clean and large-sized flakes. An electrochemical approach has been developed recently to produce single-layer $\mathrm{MoS}_{2}$ and $\mathrm{WS}_{2}$ flakes; however, the upward scaling of this process is limited by volumetric resistance in battery-type cells $^{30}$. In such electrochemical cells, $10 \%$ acetylene black nanoparticles are typically added to the host materials to reduce volumetric resistance but this creates one problem: carbon nanoparticles are mixed with LTMDs and these contaminants are hard to be dislodged from the surface. Similar to the research directions in solution-processed graphene, there is a clear need to explore new chemistry to make high-quality single-layer LTMDs in high yield.

The intercalation of lithium into layered molybdenum disulphide may be described as an ion-electron transfer topotactic reaction. In most reported papers, organolithium reagents were used as intercalating agents because of its solubility in a wide range of solvents and the formation of stoichiometric $\mathrm{LiMoS}_{2}$ ternary products ${ }^{26-29}$. Compared with $\mathrm{Li}$ ions, other alkali ions such as $\mathrm{Na}$ or $\mathrm{K}$ were less commonly used in exfoliation chemistry. The ionic radii of $\mathrm{Na}$ and $\mathrm{K}$ are several times larger than that of $\mathrm{Li}$ ions, which means that in principle these ions can expand the lattice in the $c$-axis direction to a larger extent. More importantly, $\mathrm{Na}$ and $\mathrm{K}$ intercalation compounds react more violently with water than $\mathrm{Li}$ compounds, implying that single-layer TMDs should be exfoliated more efficiently. Intercalation of $\mathrm{Na}$ and $\mathrm{K}$ can also produce different structural and electronic effect compared with $\mathrm{Li}$ due to different coordinative complexation by the host. In $\mathrm{Li}_{\mathrm{x}} \mathrm{MX}_{\mathrm{n}}, \mathrm{Li}$ is always octahedrally coordinated; however, $\mathrm{K}$ and $\mathrm{Na}$ can occupy octahedral or trigonal prismatic site. This has important implications electronically due to the metallic to semiconductor properties transition in these compounds. Metal electride solution consisting of $\mathrm{Na}$ in concentrated liquid ammonia can be a powerful reducing agent, except that ammonia molecule has a tendency to coordinate with Mo and displace $S$, this can result in decomposition and segregation of Mo nanoparticles ${ }^{31,32}$. In spite of numerous reports that discussed the exfoliation of LTMDs, few of these methods can meet the demand of producing high yield, high purity and large-sized flakes ${ }^{33,34}$.

Our search for alkali metal adducts lead us to naphthalene, which forms intensely coloured compounds with alkali metals. In sodium naphthalenide $\left(\mathrm{Na}^{+} \mathrm{C}_{10} \mathrm{H}_{8}^{-}\right)$, for example, the metal transfers an electron to the aromatic system to produce a radical anion which has strong reducing properties. Although sodium naphthalenide was first investigated in 1936 by Scott et al. ${ }^{35}$, the synthetic utility of this alkali metal adduct has not been fully explored. It is interesting to consider whether single-layer LTMDs can be produced by reacting $\mathrm{MoS}_{2}$ with various alkali metal naphthalenide adduct in a radical anion solution.

Motivated thus, we prepare naphthalenide adducts of $\mathrm{Li}$, $\mathrm{K}$ and $\mathrm{Na}$ and compare the exfoliation efficiency and quality of $\mathrm{MoS}_{2}$ generated. Using a two-step expansion and intercalation method, we report the production of high-quality single-layer $\mathrm{MoS}_{2}$ flake sheets with unprecedentedly large flake size, that is, up to $400 \mu \mathrm{m}^{2}$ when the $\mathrm{Na}$ adduct was intercalated. Single-layer $\mathrm{MoS}_{2}$ inks prepared by this method could be directly jet-printed on a wide range of substrate.

\section{Results}

Production of LTMDs. Figure 1 shows the schematic diagram of the processing steps involved in obtaining well-dispersed samples of LTMDs. First, bulk $\mathrm{MoS}_{2}$ crystals (or powders) are expanded by reacting with hydrazine $\left(\mathrm{N}_{2} \mathrm{H}_{4}\right)$ in hydrothermal condition (Fig. 1a). The expansion mechanism can be explained by a redoxrearrangement model in which part of the $\mathrm{N}_{2} \mathrm{H}_{4}$ is oxidized to $\mathrm{N}_{2} \mathrm{H}_{5}^{+}$upon intercalation. The intercalated $\mathrm{N}_{2} \mathrm{H}_{5}^{+}$is not thermally stable and will be decomposed to $\mathrm{N}_{2}, \mathrm{NH}_{3}$ and $\mathrm{H}_{2}$ upon heating the intercalated $\mathrm{MoS}_{2}$ films at high temperature. Decomposition and gasification of intercalated $\mathrm{N}_{2} \mathrm{H}_{4}$ molecules expands the $\mathrm{MoS}_{2}$ sheets by more than 100 times compared to its original volume. In a second step, the expanded $\mathrm{MoS}_{2}$ crystal is intercalated by alkali naphthalenide solution (Fig. 1b). Finally the intercalated $\mathrm{MoS}_{2}$ is exfoliated by dipping in ultrasonicated water operated at low power to avoid fragmentation of the sheets. A black suspension consisting predominantly of $90 \%$ single-layer $\mathrm{MoS}_{2}$ can be obtained after centrifugation and decanting the supernatant (Supplementary Fig. S1). The generic applicability of this method has been tested successfully on a wide range of LTMDs, which includes the high yield exfoliation of monolayer $\mathrm{TiS}_{2}, \mathrm{TaS}_{2}$, and $\mathrm{NbS}_{2}$, as well as few layer (2-4) diselenide $\mathrm{TiSe}_{2}$, $\mathrm{NbSe}_{2}$, and $\mathrm{MoSe}_{2}$ (Supplementary Fig. S2).

Intercalation and exfoliation. XRD measurements were done on freshly intercalated, hermetically sealed samples after drying in argon and vacuum. The position and intensity of the (002) peak originating from the hexagonal $2 \mathrm{H}-\mathrm{MoS}_{2}$ can be used to judge the extent of intercalation and exfoliation. In freshly intercalated samples, the (002) peaks of the pristine $\mathrm{MoS}_{2}$ is shifted completely toward lower angles, indicating expansion of the lattice along the $c$-axis and formation of stoichiometric ternary compounds (Fig. 2). The spacing between adjacent layers in the expanded lattice of the each intercalated phase are $\mathrm{K} c / 2=7.92 \AA$, $\mathrm{Na} c / 2=7.05 \AA$ and $\mathrm{Li} c / 2=6.18 \AA$, respectively. The increase in the interlayer distance as a result of the intercalation is $\Delta c / 2=$ (c $\left./ 2-c-c_{0} / 2\right), \mathrm{K} \quad \Delta c / 2=1.88 \AA, \mathrm{Na} \Delta c / 2=1.01 \AA$ and $\mathrm{Li}$ $\Delta c / 2=0.14 \AA$. It is worth noting that these $\Delta c / 2$ values are much smaller than that of naphthalene-intercalated $\mathrm{MoS}_{2}$ produced by an exfoliating-restacking process, which indicates that the organic 

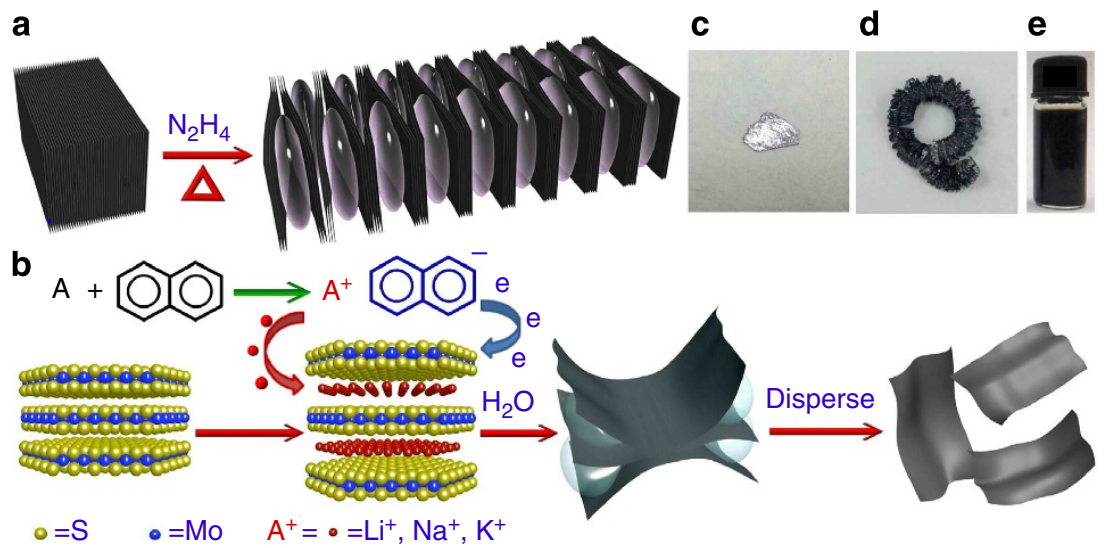

Figure 1 | Schematic of fabrication processes. (a) Bulk $\mathrm{MoS}_{2}$ is pre-exfoliated by the decomposition products of $\mathrm{N}_{2} \mathrm{H}_{4}$. (b) Pre-exfoliated $\mathrm{MoS}_{2}$ reacts with $\mathrm{A}^{+} \mathrm{C}_{10} \mathrm{H}_{8}^{-}$to form an intercalation sample, and then exfoliates to single-layer sheets in water. (c) Photograph of bulk single-crystal MoS ${ }_{2}$,

(d) photograph of pre-exfoliated $\mathrm{MoS}_{2}$ (e) photograph of Na-exfoliated single-layer $\mathrm{MoS}_{2}$ dispersion in water.

a

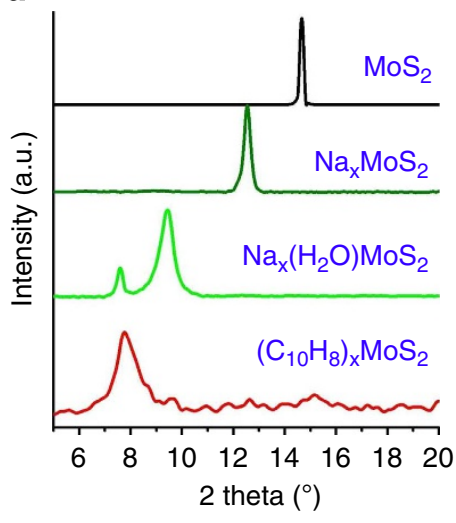

C

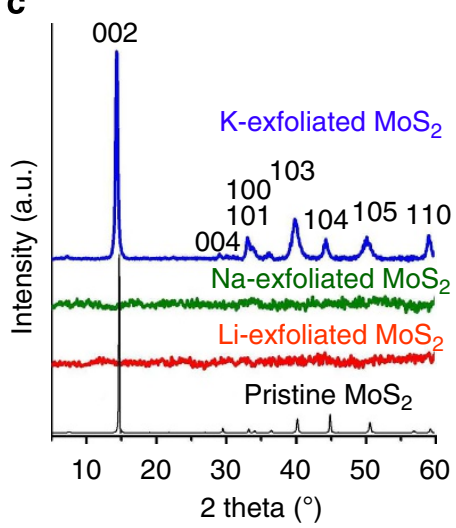

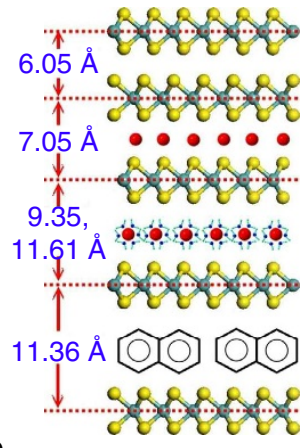

d

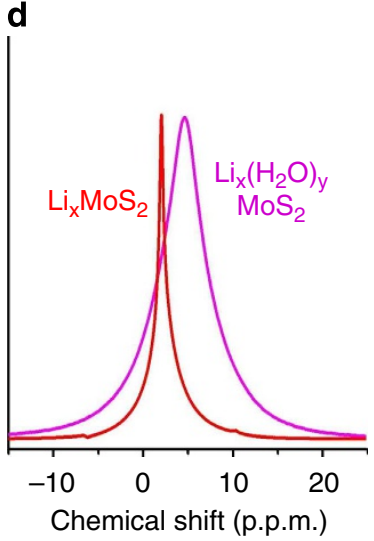

b
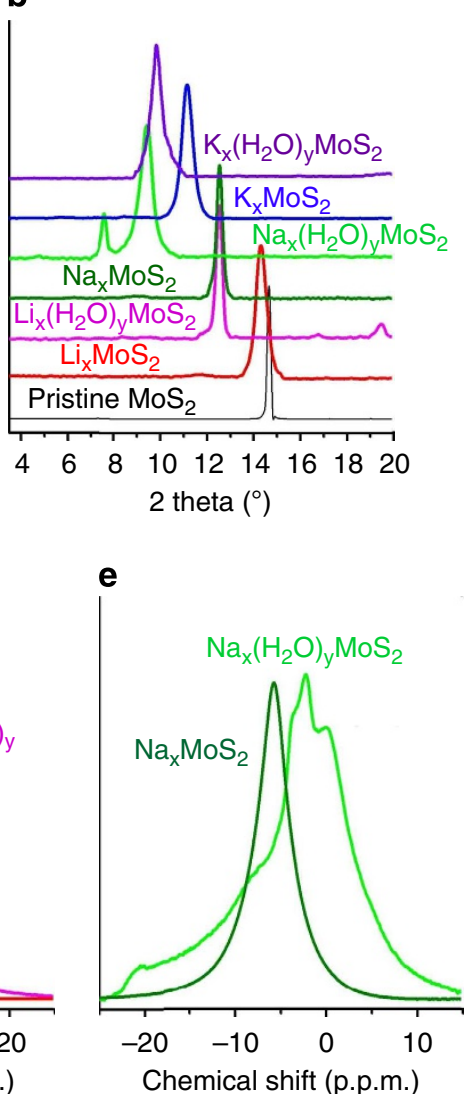

Figure 2 | Characterization of intercalated and exfoliated $\mathbf{M o S}_{\mathbf{2}}$. (a) XRD pattern and schematic of pristine MoS, $\mathrm{Na}$-intercalated MoS $\mathrm{S}_{2}$ after exposure of $\mathrm{Na}$-intercalated $\mathrm{MoS}_{2}$ to the ambient for 3 days, exfoliated-and-restacked naphthalene-intercalated MoS . (b) Li-, Na- and K-intercalated $\mathrm{MoS}_{2}$, after exposure of intercalated sample to the ambient for 3 days. (c) Li-, Na- and K-exfoliated MoS 2 without any annealing. (d) Solid-state ${ }^{7}$ Li NMR spectra of $\mathrm{Li}_{x} \mathrm{MoS}_{2}$ and $\mathrm{Li}_{x}\left(\mathrm{H}_{2} \mathrm{O}\right)_{y} \mathrm{MoS}_{2}$, (e) Solid-state ${ }^{23} \mathrm{Na}$ NMR spectra of $\mathrm{Na}_{x} \mathrm{MoS}_{2}$ and $\mathrm{Na}_{x}\left(\mathrm{H}_{2} \mathrm{O}\right)_{y} \mathrm{MoS}_{2}$.

anion is not intercalated in the $\mathrm{MoS}_{2}$ crystal. Fig. $2 \mathrm{~b}$ shows that after three days of storage in wet air (humidity around $\left.27.5 \mathrm{~g} \mathrm{~m}^{-3}\right)$, the intensity of the (002) peaks is reduced and they shift toward smaller angles $(\mathrm{K} c / 2=8.99 \AA, \mathrm{Na} c / 2=9.35$ and $11.61 \AA$ and $\mathrm{Li} c / 2=7.08 \AA$ ). This additional shift is ascribed to the continuous hydration of the intercalated ions, leading to further lattice expansion.
The intercalated compounds are exfoliated by hydration, and the exfoliated sheets are dried into powder form and characterized by XRD (Fig. 2c). In the case of K-intercalated $\mathrm{MoS}_{2}$, peaks due to the intercalated host compound as well as restacked (002) peak of $\mathrm{MoS}_{2}$ show significant intensities, which reflects incomplete exfoliation. However the (002) peaks vanish completely in the exfoliated Na-intercalated and Li-intercalated 

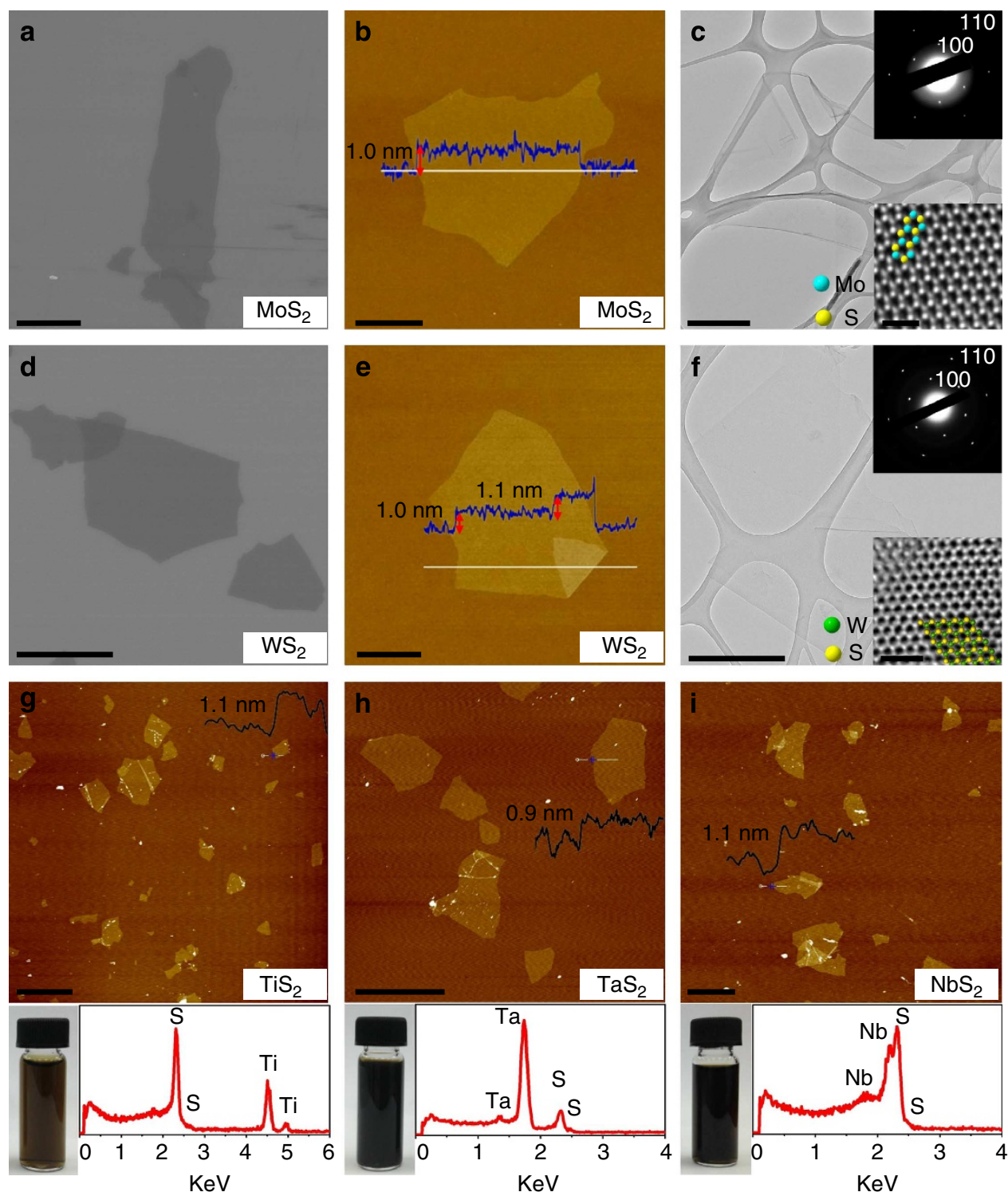

Figure 3 | Mophology characterization of LTMDs. (a) SEM images of Na-exfoliated single-layer MoS 2 . (b) AFM images of Na-exfoliated single-layer $\mathrm{MoS}_{2}$. (c) TEM images of Na-exfoliated single-layer $\mathrm{MoS}_{2}$, insets are the corresponding SAED and aberration-corrected HRTEM images. (d) SEM images of $\mathrm{Na}$-exfoliated single-layer $\mathrm{WS}_{2}$. (e) AFM images of Na-exfoliated single-layer $\mathrm{WS}_{2}$. (f) TEM images of Na-exfoliated single-layer WS $\mathrm{W}_{2}$, insets are the corresponding SAED and aberration-corrected HRTEM images. (g) AFM images TiS 2 , and corresponding EDS and photograph of dispersion in water. (h) AFM images of $\mathrm{TaS}_{2}$. (i) AFM images of $\mathrm{NbS}_{2}$. (g-i) give average thickness of $\sim 1 \mathrm{~nm}$, confirming that single-layer is successfully produced by our method. Scale bars in (a) is $10 \mu \mathrm{m}$, in $(\mathbf{b}, \mathbf{d}, \mathbf{g}, \mathbf{h})$ are $5 \mu \mathrm{m}$, in $(\mathbf{c}, \mathbf{e})$ are $1 \mu \mathrm{m}$, in (f) is $500 \mathrm{~nm}$, in (i) is $2 \mu \mathrm{m}$, in inner images of $(\mathbf{c}, \mathbf{f})$ are $1 \mathrm{~nm}$.

samples, which is a signature of complete exfoliation ${ }^{29,30}$. After annealing at $150{ }^{\circ} \mathrm{C}$ to remove water, weak (002) peaks recover in the $\mathrm{Na}$ and Li-exfoliated samples due to limited degree of restacking (Supplementary Fig. S3). Solid-state NMR is used to study the local coordination environments of the alkali metal cations before and after hydration (Fig. 2d,e). The central peaks in freshly intercalated $\mathrm{MoS}_{2}$ are sharp and symmetrical indicating a highly uniform chemical environment for most intercalated cations. After hydration, both central peaks of $\mathrm{Li}^{7}$ and $\mathrm{Na}^{23}$ solid NMR are broadened and shifted to lower frequencies, which can be attributed to dynamic processes or complex coordination environment with $\mathrm{H}_{2} \mathrm{O}$ molecules.

Morphology characterization. The uniformity and size distribution of the single-layer LTMDs sheets are examined using scanning electron microscopy (SEM) (Fig. 3a,d). One remarkable result is that $80 \%$ of the single-layer $\mathrm{MoS}_{2}$ sheets has lateral widths of around $10 \mu \mathrm{m}$, this about 10 times larger than solutionexfoliated flakes reported using $n$-butyl lithium methods ${ }^{28,30}$. As shown in Fig. 3a, a typical single-layer $\mathrm{MoS}_{2}$ flake has a surface area of $400 \mu \mathrm{m}^{2}$. When the same intercalation-and-exfoliation process is performed on $\mathrm{WS}_{2}$ crystals, $80 \%$ of the exfoliated flakes obtained are determined to be single layers with lateral dimensions between 3 and $10 \mu \mathrm{m}$, which essentially match the grain size of $\mathrm{WS}_{2}$ powder before exfoliation (Fig. 3d). To assess the effectiveness of this method, the exfoliation yield in terms of micron-sized flakes is compared with the commonly used exfoliating agent $n$-butyl lithium on the same starting materials, the results show that only submicron-sized flakes can be generated using the latter (Supplementary Figs S4-S6).

The thickness of the exfoliated sheets is characterized by atomic force microscopy (AFM). Fig. 3b,e show typical tapping mode AFM images of exfoliated $\mathrm{MoS}_{2}$ and $\mathrm{WS}_{2}$ deposited on a 

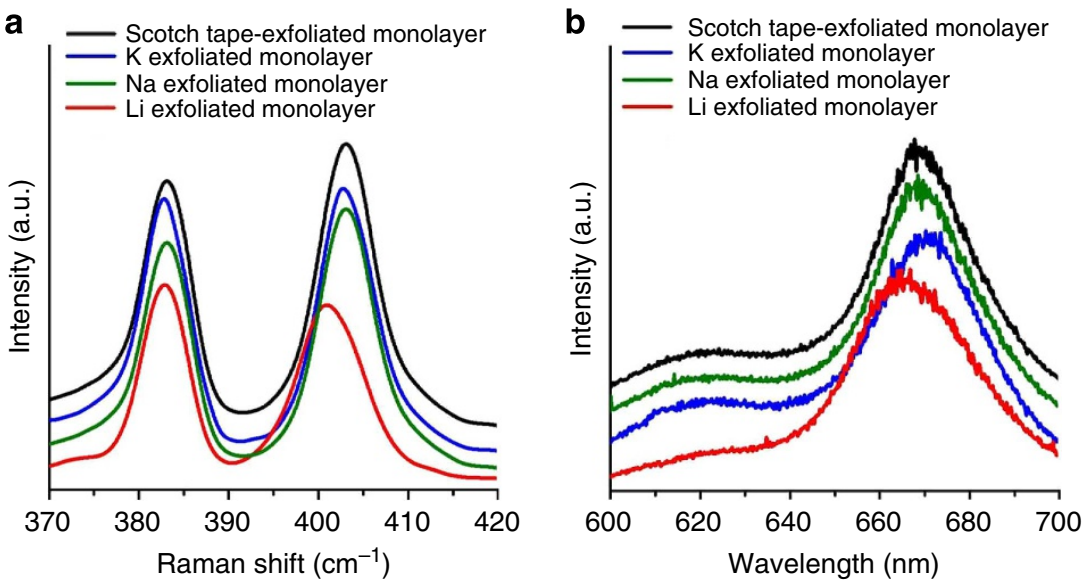

Figure 4 | Raman and photoluminescence spectrum of $\mathbf{M o S}_{\mathbf{2}}$ (a) Raman spectra of scotch-tape-exfoliated single-layer MoS $\mathrm{S}_{2}$ and Na-, $\mathrm{K}-$ and Li-exfoliated single-layer $\mathrm{MoS}_{2}$ deposited on $\mathrm{Si} / \mathrm{SiO}_{2}$ substrate. (b) Photoluminescence spectrum of scotch-tape-exfoliated single-layer $\mathrm{MoS}$, and Na-, $\mathrm{K}$ - as well as Li-exfoliated single-layer $\mathrm{MoS}_{2}$ nanosheet deposited on $\mathrm{Si} / \mathrm{SiO}_{2}$ substrate.

$\mathrm{SiO}_{2} / \mathrm{Si}$ substrate by spin-coating. The average topographic height is around $1 \mathrm{~nm}$, which agrees with typical height of a single-layer $\mathrm{MoS}_{2}$ (between 0.6 and $\left.1.0 \mathrm{~nm}\right)^{30}$. Statistical analysis of 100 flakes produced by the three different alkali metal adduct reveal 20, 90 , and $80 \%$ of the flakes to be monolayer for $\mathrm{K}_{\mathrm{x}} \mathrm{MoS}_{2}, \mathrm{Na}_{\mathrm{x}} \mathrm{MoS}_{2}$, and $\mathrm{Li}_{\mathrm{x}} \mathrm{MoS}_{2}$, respectively (Supplementary Fig. S7). Although $\mathrm{K}_{\mathrm{x}} \mathrm{MoS}_{2}$ reacts more violently with water than $\mathrm{Na}_{\mathrm{x}} \mathrm{MoS}_{2}$ and $\mathrm{Li}_{\mathrm{x}} \mathrm{MoS}_{2}$, its large ionic radius precludes full intercalation. Intercalating $\mathrm{WS}_{2}$ with sodium naphthalenide also produces single-layer $\mathrm{WS}_{2}$ flakes with a yield of $\sim 90 \%$.

Transmission electron microscopy (TEM) and selected area electron diffraction (SAED) were performed on the exfoliated flake suspended on a lacey carbon TEM grid (Fig. 3c,f). The SAED patterns of exfoliated $\mathrm{MoS}_{2}$ and $\mathrm{WS}_{2}$ exhibit high crystallinity (the inset in Fig. 3c,f, Supplementary Fig. S8), as judged from the characteristic honeycomb lattice. From XPS analysis, the Na-exfoliated $\mathrm{MoS}_{2}$ film shows Mo $3 d$ peaks with peak position and width characteristic of the $2 \mathrm{H}$ phase $^{28}$ (Supplementary Figs S9-S11).

Optical characterization. The Raman spectra of the exfoliated $\mathrm{MoS}_{2}$ flakes were recorded using a 532-nm excitation line (Fig. 4a and Supplementary Fig. S12). For Na-exfoliated samples, the $E_{2 \mathrm{~g}}^{1}$ phonons stiffen with decreasing number of layers and a blue shift of the peak from $380 \mathrm{~cm}^{-1}$ of the thick layers to $383 \mathrm{~cm}^{-1}$ of monolayer $\mathrm{MoS}_{2}$ occurs. On the other hand, $A_{1 \mathrm{~g}}$ phonons soften with decreasing number layers, giving rise to a red shift from $407 \mathrm{~cm}^{-1}$ in the bulk material to $403 \mathrm{~cm}^{-1}$ in the monolayer. The Raman signature obtained is consistent with that of mechanically exfoliated single-layer $\mathrm{MoS}_{2}$ (ref. 36). The corresponding Raman peaks in Li-exfoliated $\mathrm{MoS}_{2}$ are much broader, which can be due to slight doping and possible presence of defects.

Single-layer $\mathrm{MoS}_{2}$ exhibits a unique signature in its optical spectrum in the form of photoluminescence $(\mathrm{PL})$ due to the transition from an indirect to a direct-bandgap semiconductor (Supplementary Fig. S13). MoS 2 appears in two distinct symmetry: $2 \mathrm{H}$ (trigonal prismatic $\mathrm{D} 3 \mathrm{~h}$ ) and $1 \mathrm{~T}$ (octahedral Oh) phases. The $2 \mathrm{H}$ phase is semiconducting while $1 \mathrm{~T}$ is metallic (Supplementary Note 1). In this work, PL can be observed on the exfoliated flakes after a brief bake at $200^{\circ} \mathrm{C}$ to transform it to the $2 \mathrm{H}$ phase. As shown in Fig. $4 \mathrm{~b}$, the PL spectrum of a Naexfoliated single-layer $\mathrm{MoS}_{2}$ exhibits a peak centred at $668 \mathrm{~nm}$
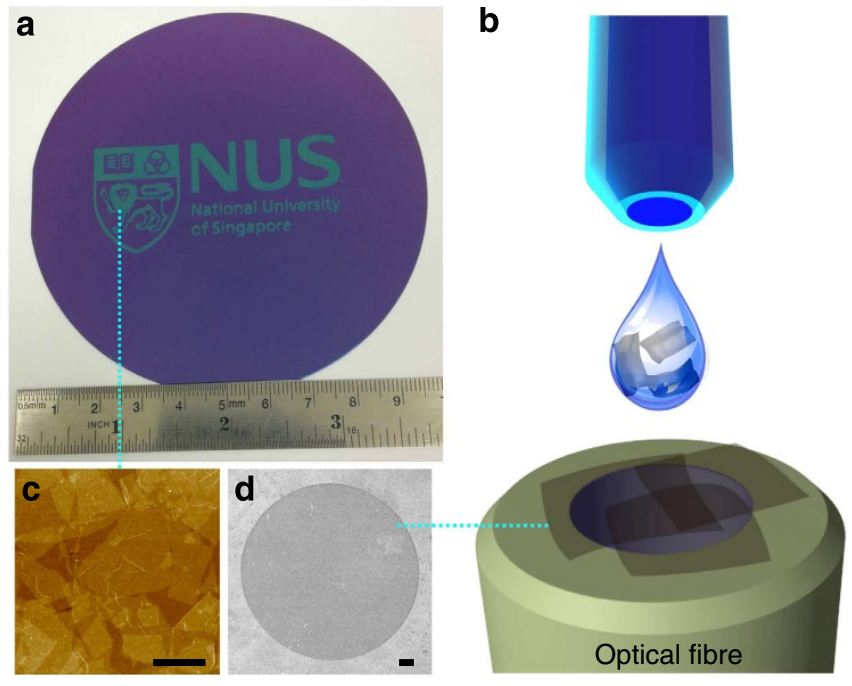

Figure $\mathbf{5}$ | Inkjet printing of $\mathbf{M o S}_{\mathbf{2}}$. (a) Wafer-scale $\mathrm{MoS}_{2}$ pattern jetprinting. Large area, continuous and highly uniform $\mathrm{MoS}_{2}$ thin film pattern were directly printed on a 4 -inch $\mathrm{Si} / \mathrm{SiO}_{2}$ wafer by inkjet printing.

(b) Schematic showing the printing of $\mathrm{MoS}_{2}$ thin film on the optical fibre pigtail. (c) AFM image of $\mathrm{MoS}_{2}$ thin film, showing a thickness of 1-4 layers. (d) SEM image of $\mathrm{MoS}_{2}$ thin film-coated optical fibre pigtail. Scale bar is $5 \mu \mathrm{m}$ in (c,d).

$(1.86 \mathrm{eV})$ with a shoulder at $623 \mathrm{~nm}(1.99 \mathrm{eV})$, which agrees with excitonic peaks arising from the $\mathrm{K}$ point of the Brillouin zone. The PL peak position and peak width is consistent with mechanically cleaved monolayer $\mathrm{MoS}_{2}$ (ref. 3). The Liexfoliated monolayer sample shows a weaker PL peak, this reflects either slight doping or the presence of defects in it. The chemical purity of the Na-exfoliated $\mathrm{MoS}_{2}$ flakes is verified by energy-dispersive X-ray spectroscopy (Supplementary Fig. S14) and its electrical property is evaluated in the form of a field effect transistor (Supplementary Fig. S15 and Supplementary Note 2). The field effect mobility of monolayer $\mathrm{MoS}_{2}$ flake is measured to be in the range of $1-8 \mathrm{~cm}^{2} /(\mathrm{V} \times \mathrm{s})$ while that of few layer $\mathrm{MoS}_{2}$ flakes are in the range of $20-80 \mathrm{~cm}^{2} /(\mathrm{V} \times \mathrm{s})$, which are comparable with those of field effect transistor made from mechanically exfoliated $\mathrm{MoS}_{2}$ flakes ${ }^{37}$. 
Inkjet printing. Inkjet printing is highly promising for the highthroughput deposition of micron-sized patterns by virtue of its speed, low cost, additive and direct writing capability ${ }^{38}$. The good dispersion and high viscosity of our $\mathrm{MoS}_{2}$ dispersion render it highly suitable for jet-printing. The ink is made from $0.02 \mathrm{mg} \mathrm{ml}^{-1} \quad \mathrm{MoS}_{2}$ fully dispersed in ethanol/water $(2: 1$ volume) solution (viscosity $2.64 \mathrm{cP}$ and surface tension $34.3 \mathrm{mN} \mathrm{m}^{-1}$ ). To print high-resolution patterns and uniform films, $10-\mu \mathrm{m}$ diameter printer nozzle is selected, and the wafers are heated to $60^{\circ} \mathrm{C}$ before printing (Supplementary Figs S16, S17 and Supplementary Note 3 ). Owing to the moderate surface energy of the ink, $\mathrm{MoS}_{2}$ inks can be directly printed on plastic, $\mathrm{SiO}_{2}$, glass and optical fibre pigtail (Fig. 5a-d) without any chemical/physical modification. Figure 5 a shows the word 'NUS' printed directly using the $\mathrm{MoS}_{2}$ flakes. The AFM and SEM characterization of the $\mathrm{MoS}_{2}$ printed thin film show that the printed $\mathrm{MoS}_{2}$ film is continuous and uniform with a sheet thickness of 2-3 layers, as shown in Fig. $5 c, d$.

\section{Discussion}

In summary, we have explored the use of metal naphthalenide for the intercalation-exfoliation of metal chalcogenides $\left(\mathrm{MoS}_{2}\right.$ and $\mathrm{WS}_{2}$ ) and obtained high-efficiency exfoliation of micron-sized monolayer sheet (widths in the range of $5-10 \mu \mathrm{m}$ ). The size distribution of the flake is much better than exfoliation using organolithium salts ( $n$-butyl lithium). This can be related to a reduced chemical reaction of the radical anion $\left(\mathrm{C}_{10} \mathrm{H}_{8}^{-}\right)$with the host material, and the fact that we apply a pre-expansion procedure with hydrazine to facilitate the efficient intercalation of the metal cations. Evidence from XRD and NMR shows the existence of highly ordered ternary phase after cation intercalation. In terms of exfoliation efficiency, sodium naphthalenide $\left(\mathrm{Na}^{+} \mathrm{C}_{10} \mathrm{H}_{8}^{-}\right)$produces higher quality monolayer flake than its lithium and potassium counterparts. This work contributes a high-yield chemical processing method for producing highquality $2 \mathrm{D}$ chalcogenide monolayers with direct relevance to printable photonics.

\footnotetext{
Methods

Pre-expansion of $\mathbf{M o S}_{\mathbf{2}}$. Bulk $\mathrm{MoS}_{2}$ (1.6 g; SPI, single crystal) and $20 \mathrm{ml}$ hydrazine hydrate (Aldrich, 98\%) are sealed in an autoclave and heated at $130{ }^{\circ} \mathrm{C}$ for $48 \mathrm{~h}$. The expanded $\mathrm{MoS}_{2}$, which has a worm-like appearance, is washed three times by water and dried at $120^{\circ} \mathrm{C}$ for $10 \mathrm{~h}$.
}

Intercalation of $\mathbf{M o S}_{\mathbf{2}}$. Na ( $0.69 \mathrm{~g}$; Aldrich) (for $\mathrm{K}$ or $\mathrm{Li}$, we used $1.08 \mathrm{~g}$ or $0.21 \mathrm{~g}$, respectively), $1.92 \mathrm{~g}$ naphthalene (aldrich) and $80 \mathrm{ml}$ anhydrous tetrahydrofuran (THF) (Aldrich, fresh redistilled by $\mathrm{Na}$ ) are stirred for $2 \mathrm{~h}$ in ice-water bath in argon atmosphere. Pre-expanded $\mathrm{MoS}_{2}$ powder $(1.6 \mathrm{~g})$ is added to the dark blue solution and the mixture is further stirred for $5 \mathrm{~h}$. After reaction, the product is washed five times by anhydrous THF. The procedures are similar for both $\mathrm{K}$ and $\mathrm{Li}$.

Intercalation process for $\mathrm{WS}_{2}$ is similar except that $2.48 \mathrm{~g}$ of $\mathrm{WS}_{2}$ is used with the same amount of reagent above.

Caution: $\mathrm{Li}_{\mathrm{x}} \mathrm{MoS}_{2}$ will self-heat in air, $\mathrm{Na}_{\mathrm{x}} \mathrm{MoS}_{2}$ will self-ignite in air and $\mathrm{K}_{\mathrm{x}} \mathrm{MoS}_{2}$ will self-explode in air.

Exfoliation. Distilled water $(100 \mathrm{ml})$ is added to the intercalated sample. The mixture is sonicated in a low-power sonic bath $(60 \mathrm{~W})$ for $30 \mathrm{~min}$ to form a homogeneous suspension. The mixture is centrifuged at 8,000 r.p.m. for $15 \mathrm{~min}$ for several cycles to remove excess impurity, and then at 1,000 r.p.m. for $15 \mathrm{~min}$ in the last cycle.

Equipment. The following equipment were used: Raman (Alpha 300R), PL (equipped on Raman), SEM (Jeol JSM-6701F), AFM (Dimension Fast Scan), XPS (SPECS), UV-VIS-NIR (Shimadzu UV-3600), solid NMR (Bruker $400 \mathrm{MHz}$ ), powder XRD (Siemens D5005), Jet-Printer (Dimatix, 2800).

\section{References}

1. Wang, Q. H., Kalantar-Zadeh, K., Kis, A., Coleman, J. N. \& Strano, M. S Electronics and opto-electronics of two-dimensional transition metal dichalcogenides. Nat. Nanotech. 7, 699-712 (2012).

2. Mak, K. F., Lee, C., Hone, J., Shan, J. \& Heinz, T. F. Atomically thin MoS2: a new direct-gap semiconductor. Phys. Rev. Lett. 105, 136805 (2010).

3. Splendiani, A. et al. Emerging photoluminescence in monolayer MoS2. Nano Lett. 10, 1271-1275 (2010).

4. Cao, T. et al. Valley-selective circular dichroism of monolayer molybdenum disulphide. Nat. Commun. 3, 887 (2012).

5. Matte, H. S. S. et al. MoS2 and WS2 analogues of graphene. Angew. Chem. Int. Ed. 49, 4059-4062 (2010).

6. Gordon, R. A., Yang, D., Crozier, E. D., Jiang, D. T. \& Frindt, R. F. Structures of exfoliated single layers of WS2, MoS2, and MoSe2 in aqueous suspension. Phys. Rev. B 65, 125407 (2002).

7. Shi, Y. et al. Van der waals epitaxy of MoS2 layers using graphene as growth templates. Nano Lett. 12, 2784-2791 (2012).

8. Lee, Y.-H. et al. Synthesis of large-area MoS2 atomic layers with chemical vapor deposition. Adv. Mater. 24, 2320-2325 (2012).

9. Coleman, J. N. et al. Two-dimensional nanosheets produced by liquid exfoliation of layered materials. Science 331, 568-571 (2011).

10. Chhowalla, M. et al. The chemistry of two-dimensional layered transition metal dichalcogenide nanosheets. Nat. Chem. 5, 263-275 (2013).

11. Butler, S. Z. et al. Progress, Challenges, and opportunities in two-dimensional materials beyond graphene. ACS Nano 7, 2898-2926 (2013).

12. Zhan, Y., Liu, Z., Najmaei, S., Ajayan, P. M. \& Lou, J. Large-area vapor-phase growth and characterization of MoS2 atomic layers on $\mathrm{SiO}_{2}$ substrate. Small 8, 966-971 (2012).

13. Chou, S. S. et al. Ligand conjugation of chemically exfoliated $\mathrm{MoS}_{2}$. J. Am. Chem. Soc. 135, 4584-4587 (2013).

14. Novoselov, K. S. et al. Electric field effect in atomically thin carbon films. Science 306, 666-669 (2004).

15. Novoselov, K. S. et al. Two-dimensional atomic crystals. Proc. Natl Acad. Sci. USA 102, 10451-10453 (2005).

16. Park, S. \& Ruoff, R. S. Chemical methods for the production of graphenes. Nat. Nanotech. 4, 217-224 (2009).

17. Radisavljevic, B., Radenovic, A., Brivio, J., Giacometti, V. \& Kis, A. Single-layer MoS2 transistors. Nat. Nanotech. 6, 147-150 (2011).

18. Yoon, Y., Ganapathi, K. \& Salahuddin, S. How good can monolayer MoS2 transistors be? Nano Lett. 11, 3768-3773 (2011).

19. Zeng, H., Dai, J., Yao, W., Xiao, D. \& Cui, X. Valley polarization in MoS2 monolayers by optical pumping. Nat. Nanotech. 7, 490-493 (2012).

20. Mak, K. F., He, K., Shan, J. \& Heinz, T. F. Control of valley polarization in monolayer MoS2 by optical helicity. Nat. Nanotech. 7, 494-498 (2012).

21. Lee, H. S. et al. MoS2 Nanosheet phototransistors with thickness-modulated optical energy gap. Nano Lett. 12, 3695-3700 (2012).

22. Radisavljevic, B., Whitwick, M. B. \& Kis, A. Integrated circuits and logic operations based on single-Layer MoS2. ACS Nano 5, 9934-9938 (2011).

23. Lee, K. et al. Electrical characteristics of molybdenum disulphide flakes produced by liquid exfoliation. Adv. Mater. 24, 2320-2325 (2012).

24. Benavente, E., Santa Ana, M. A., Mendizabal, F. \& Gonzalez, G. Intercalation chemistry of molybdenum disulfide. Coord. Chem. Rev. 224, 87-109 (2002).

25. Golub, A. S., Zubavichus, Y. V., Slovokhotov, Y. L. \& Novikov, Y. N. Singlelayer dispersions of transition metal dichalcogenides in the synthesis of intercalation compounds. Russian Chem. Rev. 72, 123-141 (2003).

26. Py, M. A. \& Haering, R. R. Structural destabilization induced by lithium intercalation in MoS2 and related-compounds. Can. J. Phys. 61, 76-84 (1983)

27. Eda, G. et al. Coherent atomic and electronic heterostructures of single-layer MoS2. ACS Nano 6, 7311-7317 (2012).

28. Eda, G. et al. Photoluminescence from chemically exfoliated MoS2. Nano Lett. 11, 5111-5116 (2011).

29. Joensen, P., Frindt, R. F. \& Morrison, S. R. Single-layer MoS2. Mater. Res. Bull. 21, 457-461 (1986).

30. Zeng, Z. Y. et al. Single-layer semiconducting nanosheets: high-yield preparation and device fabrication. Angew. Chem. Int. Ed. 50, 11093-11097 (2011).

31. Somoano, R. B., Hadek, V. \& Rembaum, A. Alkali metal intercalates of molybdenum disulfide. J. Chem. Phys. 58, 697 (1973).

32. Zak, A. et al. Alkali metal intercalated fullerene-like $\mathrm{MS}_{2}(\mathrm{M}=\mathrm{W}, \mathrm{Mo})$ nanoparticles and their properties. J. Am. Chem. Soc. 124, 4747-4758 (2002).

33. Gordon, R. A., Yang, D., Crozier, E. D., Jiang, D. T. \& Frindt, R. F. Structures of exfoliated single layers of $\mathrm{WS}_{2}, \mathrm{MoS}_{2}$, and $\mathrm{MoSe}_{2}$ in aqueous suspension. Phys. Rev. B 65, 125407 (2000).

34. Yang, D. \& Frindt, R. F. Li-intercalation and exfoliation of $\mathrm{WS}_{2}$. J. Phys. Chem. Solids 57, 1113-1116 (1996).

35. Scott, N. D., Walker, J. F. \& Hansley, V. L. Sodium naphthalene. i. a new method for the preparation of addition compounds of alkali metals and polycyclic aromatic hydrocarbons. J. Am. Chem. Soc. 58, 2442 (1936). 
36. Li, H. et al. Optical identification of single- and few-layer MoS2 sheets. Small 8, 682-686 (2012).

37. Das, S., Chen, H., Penumatcha, A. V. \& Appenzeller, J. High performance multilayer moS2 transistors with scandium contacts. Nano Lett. 13, 100-105 (2013).

38. Zhang, L. et al. Inkjet printing high-resolution, large-area graphene patterns by coffee-ring lithography. Adv. Mater. 24, 436-440 (2012).

\section{Acknowledgements}

K.P.L. is grateful for the MOE Tier 1 grant 'Two dimensional crystals as platforms for optoelectronics (R-143-000-556-112)' and also NRF-CRP project 'Novel 2D materials with tailored properties beyond graphene (R-144-000-295-281)'. H.S.S. is grateful for a grant (code no. 2011-0031630) from the Center for Advanced Soft Electronics under the Global Frontier Research Program through the National Research Foundation funded by the Ministry of Science, ICT and Future Planning, Korea.

\section{Author contributions}

J.Z. designed the work and performed the experiments. K.P.L. conceptualized the work, analysed the data and wrote the paper. H.Z., S.D., Y.P.L., C.T.N., H.S.S., H.Y.J. and B.L. performed some characterization experiments and analysed data.

\section{Additional information}

Supplementary Information accompanies this paper at http://www.nature.com/ naturecommunications

Competing financial interests: The authors declare no competing financial interests.

Reprints and permission information is available online at http://npg.nature.com/ reprintsandpermissions/

How to cite this article: Zheng, J. et al. High yield exfoliation of two-dimensional chalcogenides using sodium naphthalenide. Nat. Commun. 5:2995 doi: 10.1038/ ncomms3995 (2014) 